\title{
Management Difficulties of Neurological Care during the COVID-19 Pandemic
}

\author{
Md. Badrul Alam \\ Joint Director \& Professor of Neurology, National Institute of Neurosciences \& Hospital, Dhaka, Bangladesh
}

Neurological diseases are the leading cause of disability worldwide. Any epidemic or pandemic can give a negative consequences for neurological patients. These patients cannot get the proper management due to over burden of the epidemic. Confinement is a part of the strategy to reduce the spread of the pandemic diseases and thus it has impacted on the provision of medical care. This is particularly relevant for neurological patients. Many patients are presented with risk factors like older age, diabetes mellitus, cardiac diseases and others causes of disability. These factors increase the chance of poor prognosis of the neurological diseases. Furthermore, these patients are usually accompanied when they attend consultations; this has become very difficult during the pandemic, affecting the traditional methods of neurological care used in consultations.

The telemedicine has become a necessity for healthcare team management and for maintaining the provision of neurological care while avoiding the transmission of SARS-CoV-2 between patients, their relatives, neurologists, nurses, and researchers. Its implementation in patient care is challenging, despite the availability of the necessary resources. However, the telemedicine has previously been proposed as a response in the event of a disaster; the issue is whether it can be applied for extended periods to serve large numbers of patients.

The situation of the pandemic gave rise to the need for adaptation of patients' access to neurological care, such as stroke programmes and in-hospital and pre-hospital code stroke protocols, and to protect the personnel attending these patients. It has also been necessary to make decisions regarding patients with stroke who also present SARS-CoV-2 infection, despite the existence of tele-stroke programmes prior to the pandemic. The response to emergency situations, the follow-up of patients with chronic but changing conditions, and the continuation of treatment provision have led to the use of tools that are previously employed only rarely, such as email and other electronic tools. The limitation of these technologies is that patients often do not use them due to older age or the characteristics of their disease or due to unavailability of the technologies. Photographs or video footage recorded by patients or their family members may enable neurologists to assess clinical changes remotely like oculomotor alterations, gait, and balance. All these methods have enabled neurology departments to maintain the provision of care and patient follow-up despite the circumstances.

Operative decisions must always be taken in accordance with the directives of hospital management, giving priority to infected patients. Nonetheless, departments are responsible for decisions regarding their own functioning. The impact of the pandemic on hospitals requires functional reorganization of departments, setting new objectives and reallocating resources and responsibilities. This process must involve the participation of members of the department to ensure they are aware of and take on specific duties. Furthermore, the impossibility of holding in-person departmental meetings has led to the implementation of new forms of coordination and liaison. The organization of neurological emergencies and admissions are particularly challenging at a time when a high number of patients are infected with the virus. The initial aim of hospitalized patients being attended by teams of physicians that rotate each week could not be maintained because neurologists, admirably, wished to support the infectious diseases and internal medicine departments. Unfortunately, some colleagues also had to be quarantined. Nonetheless, the model of rotating care teams for potentially exposed patients seems to be a reasonable approach. One basic action is to maintain the continuity of care, albeit remotely, ideally through the use of telephone consultations and electronic resources. This necessity arises from the role of social isolation in reducing the spread of the disease, which has affected medical care; however, the rapid propagation of the virus has turned healthcare facilities and transport into potential sources of infection. 
Therefore, it has become essential to centre care around telephone consultations, which have represented the central element of neurological care. These calls are technical and must be informative both for the patient and for the physician, who must make the clinical decisions based on limited information.

Due to the situation of confinement, it is nearly always possible to speak with patients immediately, and follow-up consultations are often held much more regularly than before the pandemic. Patients report high satisfaction and perceive this highly personalized care as having a positive impact on their health. Neurologists will also be aware if one of their patients is infected with the virus and will be able to contribute to decisions about the patient, whose underlying neurological condition may affect prognosis. If patients present new symptoms or progression of the neurological disease, neurologists will make the appropriate decision in each case, analyzing the risk/benefit balance of the patient visiting hospital, which should be avoided where possible. Remote follow-up involves making decisions about whether to perform or postpone routine complementary studies requiring a hospital visit, which must be assessed individually. Neurologists must also consider whether in-hospital treatments should be postponed; again, these decisions should be considered on an individual basis and account for the impact of delaying or suspending treatment on the progression of the disease and possible rebound or withdrawal effects. Neurology remains a clinical specialty. It is unthinkable that any patient should be dismissed without direct examination, even if special precautions must be taken. These scheduled patients must attend the centre alone; caregivers should not accompany them except in cases where this is demonstrably necessary for the patient's continued care. It is important to take into account the need for physical separation of physician and patient. The patients should attend consultations alone where possible. The eye fundus examination should be avoided wherever possible in order to minimize physical proximity between patient and physician. The lumbar puncture should only be performed when essential.

Despite these considerations, some patients require complex treatments or essential diagnostic tests, making hospital visits unavoidable. In these cases, new, personalized forms of consultation are needed. Patients' temperature should be taken prior to consultation, and individuals presenting fever should be seen unaccompanied and all safety protocols observed. The decision involves weighing the risk of transmission of the virus against the potential benefit of the scheduled treatment or examination. We must also seek ways of following up patients in specific circumstances and enabling direct access to the neurology department. Epileptic seizures represent a challenge when patients are in isolation. Patients are generally given action plans to follow in the event of unusual seizures and have rescue medications, which may be administered by their family. It can be also provided telephone numbers for emergency consultations to minimize trips to hospital.

[Journal of National Institute of Neurosciences Bangladesh, January 2021;7(1):1-2] 